\title{
OFDMA-PON with MQAM downlink for flexible allocation
}

\author{
HE Chao ${ }^{1,2}$, WANG Ruyan ${ }^{1,2, *}$, TAN Zefu ${ }^{3}$, and TAN Xiang'an ${ }^{4}$ \\ 1. Key Laboratory of Ubiquitous Sensing and Networking in Chongqing, Chongqing University of Posts and Telecommunications, \\ Chongqing 400065, China; 2. School of Communication and Information Engineering, Chongqing University of \\ Posts and Telecommunications, Chongqing 400065, China; 3. Key Laboratory of Signal and Information Processing, \\ Chongqing Three Gorges University, Chongqing 404100, China; 4. Operation \& Maintenance Department of \\ Wanzhou Branch, China Unicorn, Chongqing 404100, China
}

\begin{abstract}
In this paper, the 40-Gbps orthogonal frequency division multiple access (OFDMA) technology enabled by subcarrier allocation in the form of integrated architecture for the intra-cell is proposed in the downlink transmission passive broadband optical access system. The data-carrying subcarriers in the inverse fast Fourier transform/fast Fourier transform (IFFT/FFT) size of 1024 points are successfully divided into three sub-channels, in which each sub-channel has 256 useful subcarriers, by using adaptive dynamic bandwidth allocation (DBA). Taking the inherent advantages of $\mathrm{M}$-ary quadrature amplitude modulation (MQAM) modulation mechanism into account, the performance of the absolutely identical MQAM format over the different sub-channels for the downstream OFDMA-passive optical network (PON) is investigated based on the intensity modulation direct detection (IMDD) system by simulations. The results show that three parallel 4QAM or 16QAM or 64QAM OFDMA data, which are transmitted over three sub-channels, is more suitable for different sub-channel allocations, respectively. In addition, comparing with single port 4/16/64QAM OFDM over the same access system, the receiver sensitivity economizes $-0.6 \mathrm{dBm}, 0.6 \mathrm{dBm}, 4.6 \mathrm{dBm}$ at the bit error rate (BER) value of $10^{-3}$ respectively.
\end{abstract}

Keywords: orthogonal frequency division multiple access (OFDMA), passive optical network (PON), downlink transmission, dynamic bandwidth allocation (DBA).

DOI: $10.21629 / J S E E .2019 .06 .05$

\section{Introduction}

Recently, the idea of connecting everything to mobile internet such as transport, education, healthcare, manufacturing, finance and enterprise, being provided by the Global Mobile Broadband (GMBB) Forum, takes account of boosting network capacity, connecting small cells,

\footnotetext{
Manuscript received May 14, 2018.

*Corresponding author.

This work was supported by the National Natural Science Foundation of China (61771082; 61801065; 61871062), the China Scholarship Council (201908500139), and the Science and Technology Research Program of Chongqing Municipal Education Commission (KJQN201800615; KJQN201800609).
}

enhancing service experience, maximizing network potential, expanding connection opportunities and creating a better world in the upcoming future [1]. Because of the inherent balance between the data rate and the communication capacity, both mobile communication carriers and Internet-service providers will bring about high effectiveness, significant promotion as well as responsibility and pave a new way to the passive optical network (PON) based on orthogonal frequency division multiple access (OFDMA), which have a large number of things on its side, for example, high spectral efficiency (SE), steel patience to fiber dispersion introduced by pulse broadening, flexible bandwidth resource allocation, and excellent cost-effectiveness [2 - 34]. In the traditional PON method, a project, the international telecommunication union telecommunication standardization sector Gigabit PON (ITU-T GPON) series smooth evolution from GPON in 2002 to next-generation PON (NG-PON) making up of 100-Gigabit PON1 (XG-PON1) and NG-PON2, was successfully initiated by the full service access network (FSAN). On the other hand, a scheme, IEEE 802.3 Ethernet PON (EPON) series smooth evolution from EPON in 2004 to 10G-EPON in 2009 and then to NG-EPON in the form of non-return-to-zero (NRZ), Duo-Binary and 4 pulse amplitude modulation (PAM4) in 2015, was originated by the European Federation for Medical Informatics (EFMI) workgroup. At the same time, to meet the ever increasing bandwidth demands caused by big videos, big operation, big architecture and big IT from end-users, the OFDMA technique has been considered as one of the most promising broadband candidates for the next generation PON and is much taken with researchers. However, unlike the long-haul coherent detection optical OFDMA PONs, the short reach direct detection optical OFDMA PONs, especially for the downstream transmission, has a large number of advantages, for instance, more simple transceiver, lower computational complexity, lower component cost and more stable system performance [11-15]. 
Qian et al. were committed to driving the experimental demonstration of a novel OFDMA PON by offering several source-free optical network units (ONUs) and charting system framework for downstream and upstream [11]. All the same, both $40 \mathrm{~Gb} / \mathrm{s}$ and $108 \mathrm{~Gb} / \mathrm{s}$ OFDMA-PONs approaches with polarization multiplexing and direct detection in the form of 16 quadrature amplitude modulation (16QAM) were proposed and demonstrated to simplify receiver end hardware complexity [12]. Jin et al. experimentally investigated the real time OFDMA-PONs structure of reflective semiconductor optical amplifier (RSOA)based in ONU1 and distributed feedback (DFB)-based in ONU2 enabled by 32-QAM in subcarriers of 1 to 7 and 64-QAM in subcarriers of 8 to 15 modulation technologies in the intensity modulation direct detection (IMDD) broadband optical access networks OANs for the uplink transmission, respectively, and the wavelength spacing between the two adjacent ONUs was studied across the C-band [13]. In addition, the OFDMA PON bidirectional transmission with coherent detection optical line terminal (OLT) and direct detection ONUs for $21.6 \mathrm{~Gb} / \mathrm{s}$ QPSK and $14.5 \mathrm{~Gb} / \mathrm{s} 32 \mathrm{QAM}$ was realized in [14] by using carrierreuse, single-feeder and wavelength division multiplex (WDM) based on single fiber light wave centralization. He et al. verified a full-duplex optical access system with hybrid 64/16/4QAM-OFDM downlink and NRZ uplink, where bit error rate (BER) values lower than hardware decision forward error correction (HD-FEC) are obtained after $20 \mathrm{~km}$ length standard single mode fiber (SSMF) transmission [15]. Chen et al. firstly demonstrated the real-time reconfigurable 4/16/64QAM OFDM data in X-band radio over fiber (RoF) system. Moreover, after $1.5 \mathrm{~m}$ wireless and $2.26 \mathrm{~km}$ SSMF, the systems achieve the BER required by communication link, namely, HD-FEC threshold of $3.8 \times 10^{-3}[16]$.

In this paper, a $40 \mathrm{Gbps}$ three sub-channels OFDMA downlink architecture enabled by M-ary QAM (MQAM) technology is experimentally proposed and simulated over $20 \mathrm{~km}$ length SSMF transmission in IMDD PON access system, where each sub-channel has been made up of 256 successively possible sub-carriers and three sub-channels are absolutely conveyed by three identical 4QAM, 16QAM, 64QAM data streams. This project successfully employs both channel estimation (CE) and carrier phase estimation (CPE) at the receiver side to improve system performance. The best sub-channel receiving performance of 4QAM OFDMA PON is the second sub-channel, 16QAM is the first sub-channel, 64QAM is the second subchannel. In addition, the receiver sensitivity has been obviously enhanced by $-0.6 \mathrm{dBm}, 0.6 \mathrm{dBm}, 4.6 \mathrm{dBm}$ comparing with single port 4QAM, 16QAM, 64QAM OFDMA broadband OAN.
In particular, the main advantages of this design in this paper is that three different data symbols transmitting over three sub-channels can save the spectrum resource to compare the existing model.

\section{System setup}

As Fig. 1 shows, the subcarrier indexes in the range of 129 to 384,385 to 640 , and 641 to 896 are defined as the first sub-channel, the second sub-channel and the third sub-channel, respectively. It is noteworthy that the maximum possible subcarrier in the inverse fast Fourier transformation (IFFT) is 1024 , in which, the total 768 location subcarriers varying from 129 to 896 are successfully implemented to convey three useful MQAM data streams. Moreover, the number of prefix points, the size of training symbols, and the averaged OFDM power are 100, 8 and $16 \mathrm{dBm}$, respectively.

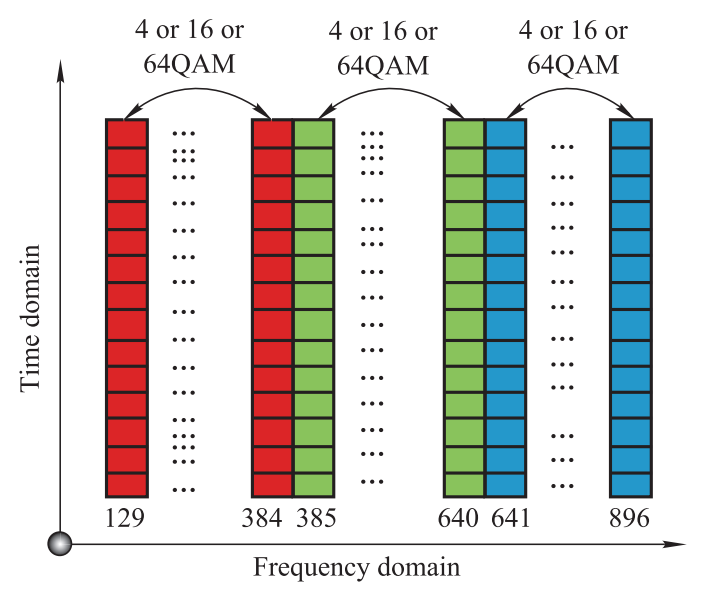

Fig. 1 Three sub-channels allocation

The setup of the three sub-channels MQAM OFDMA PON system for the downlink transmission is in reported detail in Fig. 2, which is constitutive of OLT in the transmitting central office (CO), optical fiber link between OLT and $\mathrm{ONU}$, and $\mathrm{ONU}$ in the receiving base station (BS). At the OLT, three parallel binary input data streams are generated by three pseudo random bit sequence generators (PRBSs) with the bit rate of global bit rate $\times 256 / 1024$. Each binary data stream is firstly mapped into MQAM, then OFDM modulation is executed and finally digital-toanalog converter (DAC) is performed with the sample rate of 10 Gigabit samples per second and cubic interpolation filtering to generate the MQAM OFDMA baseband electrical signal. Here, it also needs to mention that OFDM modulation will carry out a series of process, including serial/parallel conversion (S/P), IFFT, insertion cyclic prefix (CP) in the form of symbol extension in guard inter$\mathrm{val}$, and parallel/serial conversion (P/S). In the electrical to optical (E/O) convertor, MQAM OFDMA baseband electrical signal functioning as the electrical modulation sig- 
nal directly drives the dual port Mach-Zehnder modulator (DP-MZM), in which, the extinction ration (ER), switching bias voltage, switching radio frequency (RF) voltage, insertion loss, bias voltage 1 , bias voltage 2 are $30 \mathrm{~dB}, 4 \mathrm{~V}$, $4 \mathrm{~V}, 2 \mathrm{~dB}, 0 \mathrm{~V}, 2 \mathrm{~V}$, respectively. A continuous wavelength (CW) optical signal generated by the external cavity laser (ECL1), functioning as the optical carrier signal, is modulated by an intensity modulator in terms of DP-MZM, the other continuous wave optical signal generated by ECL2, without data modulation, is recombined with the MQAM OFDMA modulated baseband optical signal with the help of a $3 \mathrm{~dB}$ optical coupler (OC) after modulation. Both ECL1 and ECL2 have the exactly same value in power, line-width and initial phase, which are $4 \mathrm{dBm}, 0.1 \mathrm{MHz}$, and $0^{\circ}$, but only different in the frequency value. Then, the erbium-doped fiber amplifier (EDFA) with both $10 \mathrm{~dB}$ gain and $4 \mathrm{~dB}$ noise figure is exploited to enhance the transmission optical power and eliminate the greater additional insertion loss caused by passive optical splitter (OS) before $20 \mathrm{~km}$ long span transmission of SSMF that possesses features of the attenuation factor of $0.2 \mathrm{~dB} / \mathrm{km}$, the chromatic dispersion (CD) coefficient of $16.75 \mathrm{ps} / \mathrm{nm} / \mathrm{km}$ and the polarization mode dispersion (PMD) coefficient of $0.05 \mathrm{ps} / \sqrt{\mathrm{km}}$. Subsequently, the OS is used to split the received optical signal into two branches: one is used for downlink direct detection and another uplink seamless connection. At the ONU, the received optical MQAM
OFDMA modulated optical baseband signal is firstly filtered by Bessel optical filter (BOF1) to limit the signal bandwidth and select the center frequency, then attenuated by a variable optical attenuator (VOA) to make the received optical power (ROP) preferably meet the signal noise ratio (SNR), and finally detected by a positive intrinsic negative photodiode (PIN PD) to convert the optical signal into the electrical signal. As such, after analog to digital converter (ADC) and down-sampling, OFDM demodulation is of great importance at the receiver side, which consists of S/P, removing CP, fast Fourier transform (FFT), CE by means of training symbols, CPE by dint of pilot symbols, $\mathrm{P} / \mathrm{S}$. Without loss of generality, the generated M-ary symbol sequences will be de-mapped into binary signals as a function of the QAM sequence decoder principle. In order to perspicuously achieve the relationship between BER and ROP, BER testers are carried out to compare the transmitted binary sequence signal with the received ones. In the same BER, the single port used for transmitting the MQAM data symbol needs the lower ROP to compare the three sub-channels, in which the former occupies the subchannel index from 129 to 896, and the latter either occupies the subcarrier from 129 to 384 (first sub-channel set), or from 385 to 640 (the second sub-channel set), or from 641 to 896 (the third sub-channel set), i.e., the three sub-channel set is used to transmit the data symbol for the 4QAM, 16QAM, 64QAM in a time slot, respectively.

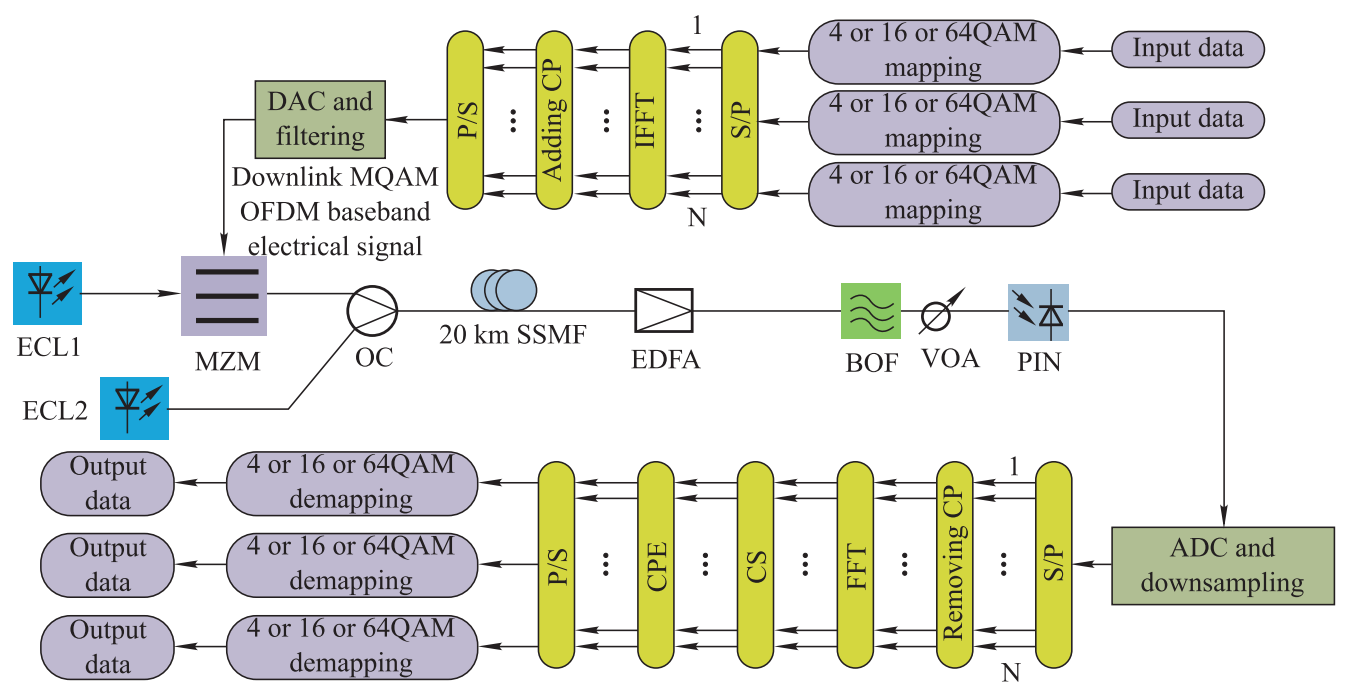

Fig. 2 System simulation for three sub-channels MQAM OFDMA in PON downlink

\section{Simulation results}

Without loss of generality, we consider OFDMA PON with MQAM downlink transmission to carry out the flexible allocation. In order to achieve the numerical results, Table 1 presents the parameter settings in our paper in detail. In addition, we adapt the Optisystem 7.0 to simulate the practical model.
It can be achieved some information that the research specialist staff are interested in from Fig. 3. As it is known that the lower level modulation format occupies more broadband frequencies while the lower level modulation with the lower constellation value, the higher bit rate and the better BER performance. Three 4QAM data, three 16QAM data and three 64QAM data make up the frequency bandwidths of $1.8 \mathrm{GHz}, 1.0 \mathrm{GHz}$ and $0.6 \mathrm{GHz}$ at 
the power changing from $-35 \mathrm{dBm}$ to $0 \mathrm{dBm}$, respectively. In order to further investigate the relationship between the BER and the ROP in the OFDMA optical access network for the downstream data, the plots of the 4QAM, 16QAM, 64QAM optical OFDMA signal composed by three subchannels after 20-km-long SSMF propagation are shown in Figs. 4-6, respectively.

Table 1 Parameter settings in the simulation

\begin{tabular}{cc}
\hline Description & Value \\
\hline Bit rate/(Gb/s) & 40 \\
IFFT size & 1024 \\
Three subchannels & $129-384,385-640,641-896$ \\
Cyclic prefix & 100 \\
Training symbol & 8 \\
Fiber length $/ \mathrm{km}$ & 20 \\
Averaged power/dBm & 16 \\
Attenuation factor $/(\mathrm{dB} / \mathrm{km})$ & 0.2 \\
CD coefficient $/(\mathrm{ps} / \mathrm{nm} / \mathrm{km})$ & 16.75 \\
PMD coefficient $/(\mathrm{ps} / \mathrm{sqrt}(\mathrm{km}))$ & 0.05 \\
\hline
\end{tabular}

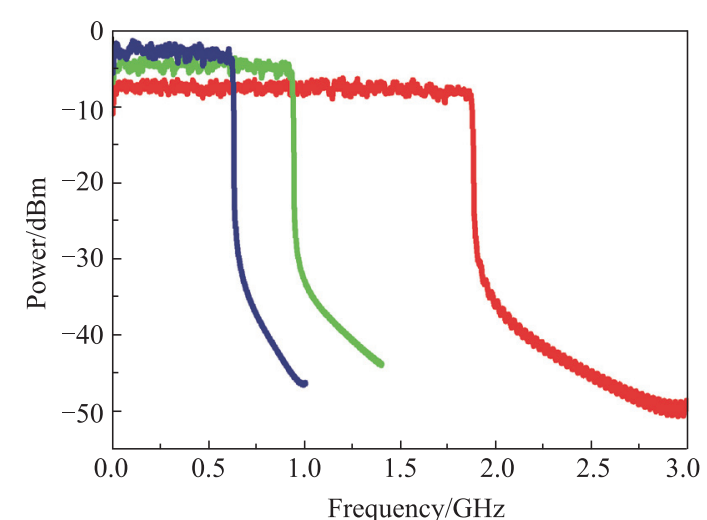

— : Three 4QAM data; — : Three 16QAM data; _ : Three 64QAM data.

Fig. 3 Power spectral density for different sub-channels

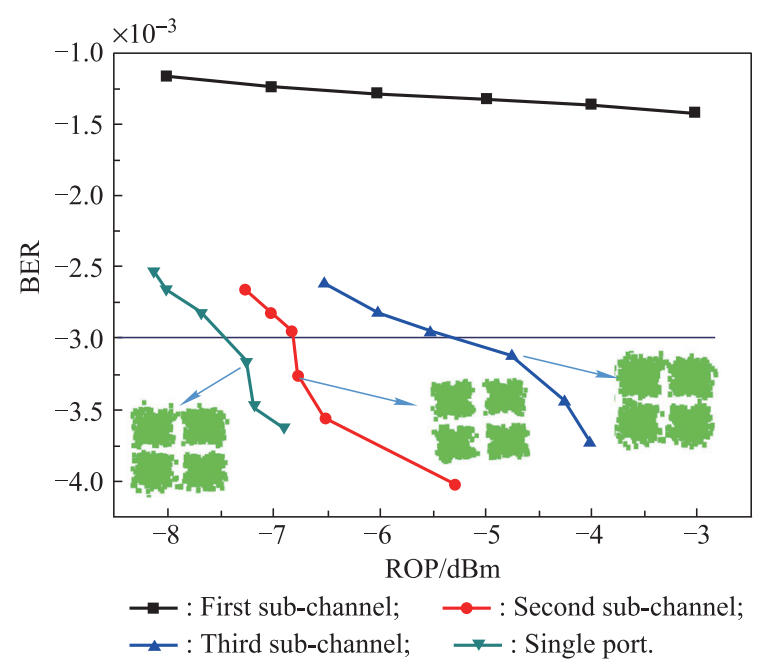

Fig. 4 BER performance versus ROP for 4QAM OFDMA downlink transmission

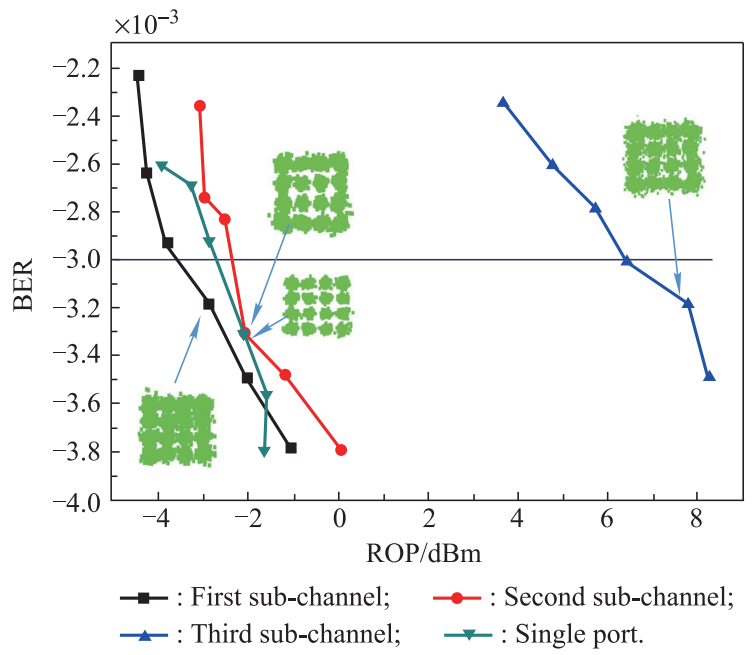

Fig. 5 BER performance versus ROP for 16QAM OFDMA downlink transmission

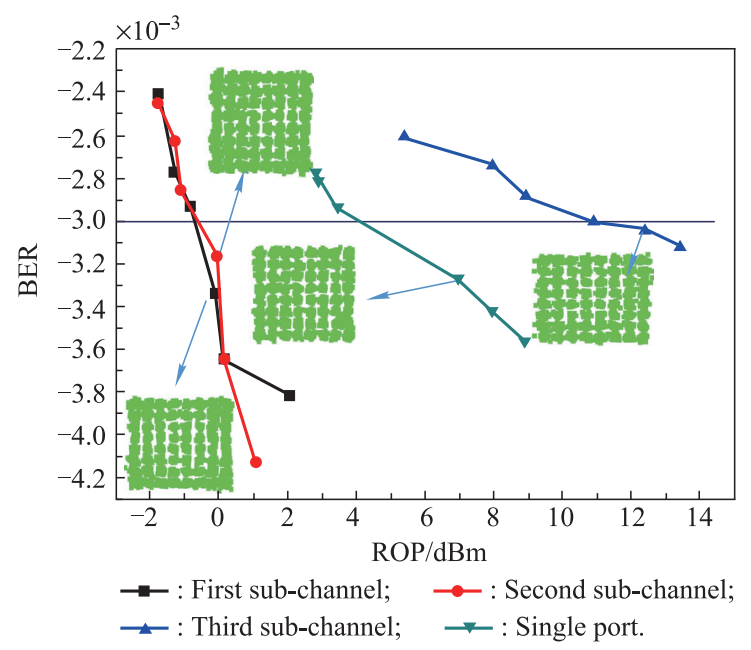

Fig. 6 BER performance versus ROP for 64QAM OFDMA downlink transmission

It is worth explaining that the subcarrier index of the single port MQAM OFDMA transmission situation is distributed in the range of 129 to 896 . In this paper, we adapt the time-division multiplexing PON (TDM-PON) to realize the dynamic resource allocation. Therefore, the three users enabled by the ONU can use the three sub-channels in a time slot to transmit the data symbol.

\subsection{Three sub-channels 4QAM OFDMA data}

To vary VOA values not only dynamically changes the ROP but also achieves appropriate BER, in which the other parameters maintain stable all the time. It can be clearly seen from Fig. 4 that the performance of the first subchannel 4QAM transmission is worse than both the second and the third sub-channels in the same simulation environment. Except for the first sub-channel, at the BER value of $10^{-3}$ the receiver sensitivities of the second sub-channel, 
the third sub-channel and the single port 4QAM OFDMA used for the direct detection system are $-6.8 \mathrm{dBm}$, $-5.3 \mathrm{dBm}$, and $-7.4 \mathrm{dBm}$, respectively. At the same time, the constellation diagrams are estimated at the BER values of $-3.25 \times 10^{-3},-3.12 \times 10^{-3}$, and $-3.15 \times 10^{-3}$, namely, the ROPs are $-6.8 \mathrm{dBm},-4.8 \mathrm{dBm}$, and $-7.2 \mathrm{dBm}$. Under the circumstances, the lower level 4QAM transmission is more suitable for the intermediate frequency (IF) band and substantially economizes the receiver sensitivity of $1.5 \mathrm{dBm}$ compared with the third subchannel.

\subsection{Three sub-channels 16QAM OFDMA data}

Similarly, the three ports 16QAM OFDMA transmission quality is successfully depicted in Fig. 5, where the ROP ranging from $-3.6 \mathrm{dBm}$ to $-2.2 \mathrm{dBm}$ for both the first, second sub-channels and the single port maintains the BER less than the threshold value of $3.0 \times 10^{-3}$. On this occasion, the third sub-channel located in high frequency (HF) band must increase the transmitted optical power (TOP) to keep normal communication, namely, the BER is inferior to the threshold value. However, the higher TOP not only raises the component cost, but also increases the power penalty. Besides the third sub-channel, the receiver sensitivities of the first, second sub-channels, and the single port are $-3.6 \mathrm{dBm},-2.4 \mathrm{dBm}$, and $-2.8 \mathrm{dBm}$, respectively. Meanwhile, the constellation diagrams are scaled at the BER values of $-3.2 \times 10^{-3}$, $-3.3 \times 10^{-3}$, and $-3.3 \times 10^{-3}$, namely, the ROPs are $-2.8 \mathrm{dBm},-2.1 \mathrm{dBm}$, and $-2.1 \mathrm{dBm}$.

\subsection{Three sub-channels 64QAM OFDMA data}

The case of 64QAM OFDMA downlink performance in the form of three ports is illustrated in Fig. 6. It is clear that the BER of the downstream data after $20 \mathrm{~km} \mathrm{SSMF}$ transmission linearly decreases from -2.44 to -4.12 by increasing the TOP and adjusting the VOA to make ROP range from $-1.7 \mathrm{dBm}$ to $1.1 \mathrm{dBm}$, and the corresponding single port and the third sub-channel are also significantly improved from $2.8 \mathrm{dBm}$ to $8.9 \mathrm{dBm}$, and from $5.4 \mathrm{dBm}$ to $13.4 \mathrm{dBm}$, respectively. Furthermore, the higher level 64QAM OFDMA transmitted over the low and medium frequency (LMF) band shows great potential. And for the single port situation, its performance falls in between the LMF and HF. It is observed in Fig. 6 that the receiver sensitivities are $-0.8 \mathrm{dBm},-0.6 \mathrm{dBm}, 11 \mathrm{dBm}$, and $4 \mathrm{dBm}$, respectively. In the meantime, the constellation diagrams are estimated at the BER values of $-3.3 \times 10^{-3},-3.2 \times 10^{-3}$, $-3.1 \times 10^{-3}$ and $-3.3 \times 10^{-3}$, namely, the ROPs are $-0.1 \mathrm{dBm},-0.1 \mathrm{dBm}, 12.4 \mathrm{dBm}$ and $6.9 \mathrm{dBm}$.

\section{Conclusions}

In this paper, we systematically design and demonstrate the OFDMA downlink for intra-cell in the PON enabled by three parallel MQAM ports, namely, three sub-channels MQAM. The 4-QAM, 16-QAM, and 64-QAM modulation schemes after $20 \mathrm{~km}$ SSMF transmission are simulated and the results of these three modulation schemes are compared. It is found that single 4-QAM gives the best performance (the lowest BER) compared to single 16-QAM and single 64-QAM when it comes to the same ROP. In simulation of each three ports MQAM, the results show that the best transmission sub-channels of 4QAM, 16QAM and 64QAM are the second, the first and the second subchannel, respectively. Compared with the traditional single port, results also show that the receiver sensitivities of the proposed 4QAM, 16QAM and 64QAM formats have been enhanced by $-0.6 \mathrm{dBm}, 0.6 \mathrm{dBm}$, and $4.6 \mathrm{dBm}$, respectively.

\section{References}

[1] YAN J, WU D, WANG H, et al. User centric content sharing based on D2D cellular networks. IEEE Trans. on Vehicular Technology, 2018, 67(11): 11208-11218.

[2] JUNG S M, MUN K H, JUNG S Y, et al. Optical-beatinduced multi-user-interference reduction in single wavelength OFDMA PON upstream multiple access systems with selfhomodyne coherent detection. Journal of Lightwave Technology, 2016, 34(11): 2804-2811.

[3] WEI C C, LIU H C, LIN C T, et al. Analog-to-digital conversion using sub-nyquist sampling rate in flexible delay division multiplexing OFDMA PONs. Journal of Lightwave Technology, 2016, 34(10): $2381-2390$.

[4] CVIJETIC N. OFDM for next-generation optical access networks. Journal of Lightwave Technology, 2012, 30(4): 384 398.

[5] CVIJETIC N, QIAN D, HU J. $100 \mathrm{~Gb} / \mathrm{s}$ optical access based on optical orthogonal frequency-division multiplexing. IEEE Communications Magazine, 2010, 48(7): 70-77.

[6] LIANG Y, SONG R, LI F, et al. Decoupled estimation of frequency-dependent IQI and channel for OFDM systems with direct-conversion transceivers. Journal of Systems Engineering and Electronics, 2017, 28(3): 435-441.

[7] JIN X Q, HUGUES S E, GIDDIMGS R P, et al. First real-time experimental demonstrations of $11.25 \mathrm{~Gb} / \mathrm{s}$ optical OFDMA PONs with adaptive dynamic bandwidth allocation. Optics Express, 2011, 19(21): $20557-20570$.

[8] ZHANG W, ZHANG C, JIN W, et al. Chaos coding-based QAM IQ-encryption for improved security in OFDMA-PON. IEEE Photonics Technology Letters, 2014, 26(19): $1964-$ 1967.

[9] ZHANG N N, ZHOU X, SHI J F, et al. 40Gbps OFDM-PON system using polarization division multiplexing for upstream transmission. Acta Photonica Sinica, 2014, 43(7): 0706018.

[10] HALBAI F, CHEN L, PARRE S, et al. Subcarrier indexpower modulated optical OFDM and its performance in IMDD PON systems. Journal of Lightwave Technology, 2016, 34(9): $2228-2234$.

[11] QIAN D, CVIJETIC N, HU J, et al. A novel OFDMA-PON architecture with source-free ONUs for next-generation optical 
access networks. IEEE Photonics Technology Letters, 2009, 17(21): $1265-1267$.

[12] QIAN D, CVIJETIC N, HU J, et al. 108Gb/s OFDMA-PON with polarization multiplexing and direct detection. Journal of Lightwave Technology, 2010, 28(4): 484-493.

[13] JIN X Q, TANG J M. Experimental investigations of wavelength spacing and colorlessness of RSOA-based ONUs in real-time optical OFDMA PONs. Journal of Lightwave Technology, 2012, 30(16): 2603-2609.

[14] AMIRALIZADEH S, NGUYEN A T, PARK C S, et al. Singlefiber lightwave centralized WDM-OFDMA-PON with colorless optical network units. Journal of Optical Communications and Networking, 2016, 8(4): 196-205.

[15] HE C, TAN Z F, SHAO Y F, et al. A full-duplex optical access system with hybrid 64/16/4QAM-OFDM downlink. Optoelectronics Letters, 2016, 12(5): $361-365$.

[16] CHEN M, XIAO X, YU J, et al. Demonstration of softwarereconfigurable real time FEC-enabled 4/16/64QAM OFDM signal transmission in an X-band RoF system. IEEE Photonics Journal, 2016, 8(2): 1-8.

[17] XIAO Y Q, WANG Z Y, CAO J, et al. Time frequency domain encryption with SLM scheme for physical-layer security in an OFDM-PON system. IEEE/OSA Journal of Optical Communications and Networking, 2018, 10(1): 46-51.

[18] GUO C J, LIANG J W, LI R. Long-reach SSB-OFDM-PON employing fractional sampling and super-nyquist image induced aliasing. Journal of Optical Communications and Networking, 2015, 7(12): 1120-1125.

[19] PENG J J, SUN Y, CHEN H S, et al. High-precision and low-complexity symbol synchronization algorithm based on dual-threshold amplitude decision for real time IMDD OFDMPON. IEEE Photonics Journal, 2019, 11(1): 1-14.

[20] WENG Z K, CHI Y C, WANG H Y, et al. 75-km long reach dispersion managed OFDM-PON at $60 \mathrm{Gbit} / \mathrm{s}$ with quasi-colorfree LD. Journal of Lightwave Technology, 2018, 36(12): $2394-2408$.

[21] LI C, HU R, LI H B, et al. Digital OFDM-PON based on deltasigma modulation employing binary IM-DD channels. IEEE Photonics Journal, 2016, 9(2): 1-7.

[22] LIU Y, YANG C C, LI H B. Cost-effective and spectrumefficient coherent TDM-OFDM-PON aided by blind ICI suppression. IEEE Photonics Technology Letters, 2015, 27(8): $887-890$.

[23] BI M H, FU X S, ZHOU X F, et al. A key space enhanced chaotic encryption scheme for physical layer security in OFDM-PON. IEEE Photonics Journal, 2017, 9(1): 1-10.

[24] SULTAN A, YANG X L, HAJOMER A A E, et al. Chaotic constellation mapping for physical-layer data encryption in OFDM-PON. IEEE Photonics Technology Letters, 2018, 30(4): $339-342$.

[25] ZHU S L, GUO Y, YIN Y J. ZTE's perspective on applying OFDM-PON in next converged optical and wireless networks. China Communications, 2015, 12(4): 50-57.

[26] KIM C H, JUNG S Y, JUNG S M, et al. All-optical virtual private network in OFDM-PON using microwave photonic filter. IEEE Photonics Technology Letters, 2016, 28(24): 2830 2833.

[27] ZHANG W, ZHANG C F, CHEN C, et al. Brownian motion encryption for physical-layer security improvement in CO-OFDM-PON. IEEE Photonics Technology Letters, 2017, 29(12): $1023-1026$.

[28] LI S S, CHENG M F, DENG L, et al. Secure strategy for OFDM-PON using digital chaos algorithm with fixed-point implementation. Journal of Lightwave Technology, 2018, 36(20): $4826-4833$.
[29] ZHANG W, ZHANG C F, CHEN C, et al. Hybrid chaotic confusion and diffusion for physical layer security in OFDMPON. IEEE Photonics Journal, 2017, 9(2): 1-10.

[30] HSU J H, YU M, WEI C C, et al. Employing hybrid subNyquist sampling rates to support heterogeneous services of varying capacity in 25Gbps DDM-OFDM-PON. IEEE Photonics Journal, 2018, 10(2): 1-8.

[31] XUE X W, JI W, HUANG K R, et al. Tunable multiwavelength optical comb enabled WDM-OFDM-PON with source-free ONUs. IEEE Photonics Journal, 2018, 10(3): $1-8$.

[32] HU Z Y, CHAN C K. A 7D hyperchaotic system-based encryption scheme for secure fast-OFDM-PON. Journal of Lightwave Technology, 2018, 36(16): 3373-3381.

[33] BI M H, ZHUO X H, FU X S, et al. Cellular neural network encryption scheme for time synchronization and CPAs resistance in OFDM-PON. IEEE Access, 2019, 7(5): 57129-57137.

[34] HU Z Y, CHAN C K. A real-valued chaotic orthogonal matrix transform-based encryption for OFDM-PON. IEEE Photonics Technology Letters, 2018, 30(16): 1455-1458.

\section{Biographies}

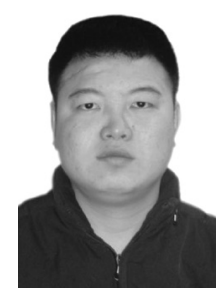

HE Chao was born in 1990. He received his B.E. and Master's degrees in School of Electronic \& Information Engineering from Chongqing Three Gorges University, Chongqing, China, in 2014 and 2017, respectively. He is currently pursuing his Ph.D. degree with Chongqing University of Posts and Telecommunications, Chongqing, China. His research interests mainly include FiWi broadband access network, network function virtualization, software defined network, mobile edge computing, and mobile cloud computing.

E-mail: hechaoctgu@163.com

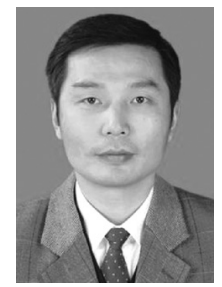

WANG Ruyan was born in 1968. He received his Ph.D. degree in 2007 from University of Electronic and Science Technology of China, and M.S. degree from Chongqing University of Posts and Telecommunications (CQUPT), China, in 1997. He is currently a full professor in CQUPT, Chongqing, China. His research interests include FiWi, network performance analysis, and multimedia information processing.

E-mail: wangry@cqupt.edu.cn

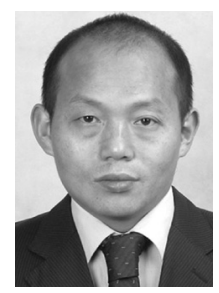

TAN Zefu was born in 1969. He received his M.S. degree majored in electromagnetic field and microwave technology from Beijing University of Posts and Telecommunications in 2002, and now he is a full professor at Chongqing Three Gorges University. Currently, his research interests include hybrid optical wireless broadband access networks. E-mail: tanzefu@163.com

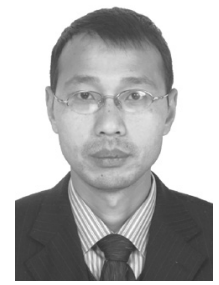

TAN Xiangan was born in 1972 . He received his M.E. degree from Hunan University in 2009. He is a senior engineer in the Operation \& Maintenance Department of Wanzhou Branch for China Unicom. His research interests include mobile software development and software engineering.

E-mail: tanxiangan@163.com 